\title{
LOW DENSITY DRIVERS OF STRONG INTERPLANETARY SHOCKS
}

\author{
A. HEWISH \\ Mullard Radio Astronomy Observatory, Cavendish Laboratory, Madingley Road, \\ Cambridge CB3 OHE, U.K.
}

\begin{abstract}
The theory that most, if not all, interplanetary shocks are caused by coronal mass ejections (CMEs) faces serious problems in accounting for the strongest shocks. The difficulties include (i) a remarkable absence of very strong shocks during solar maximum 1980 when CMEs were prolific, (ii) unrealistic initial speeds near the Sun for impulsive models, (iii) the absence of rarefaction zones behind the shocks and (iv) sustained high speed flows following shocks which are not easily explained as consequences of CME eruptions. Observations of the proton temperature near $1 \mathrm{AU}$ indicate that strong shock drivers have properties similar to high speed streams emitted by coronal holes. Eruptions of fast solar wind from coronal holes influenced by solar activity can explain the occurrence of the strongest interplanetary shocks.
\end{abstract}

\section{Introduction}

During the past decade it has become generally accepted that most, if not all, interplanetary shocks are driven by coronal mass ejections. Solar flares may sometimes also be involved but their role is no longer regarded as primary. While the cause of CMEs is not yet understood in detail it is believed to be some large-scale disruption of the coronal magnetic field leading to the release of magnetic free energy, through the mechanism of field-line reconnection, on a timescale of a few hours or less ${ }^{[1,2]}$. One difficulty with this view is the apparently anomalous behaviour of CMEs during solar maximum in 1980 when, in spite of prolific CME and flare activity, there was a remarkable absence of strong interplanetary shocks. Put quantitatively, if strong shocks are caused by CMEs, why were CMEs about five times less effective in 1980 as compared to 1978 or 1981 , bearing in mind that the properties of individual CMEs show little variation during the solar cycle?

The difficulty is too severe to be dismissed as a statistical fluke. In this paper I examine the physical characteristics of strong shocks observed by spacecraft and show that in many cases they could not have been driven impulsively by CMEs. The evidence supports a model in which the shocks were driven by sustained outflows of high-speed solar wind which need not have had an impulsive origin. A quantitative analysis of the mass flux associated with interplanetary shocks, and the relative masses of the compressed driver plasma and of the ambient wind 'swept-up' by the shock shows that the total mass of the shocked plasma in the compression region of the disturbance can sometimes be largely accounted for in terms of the swept-up 
A. HEWISH
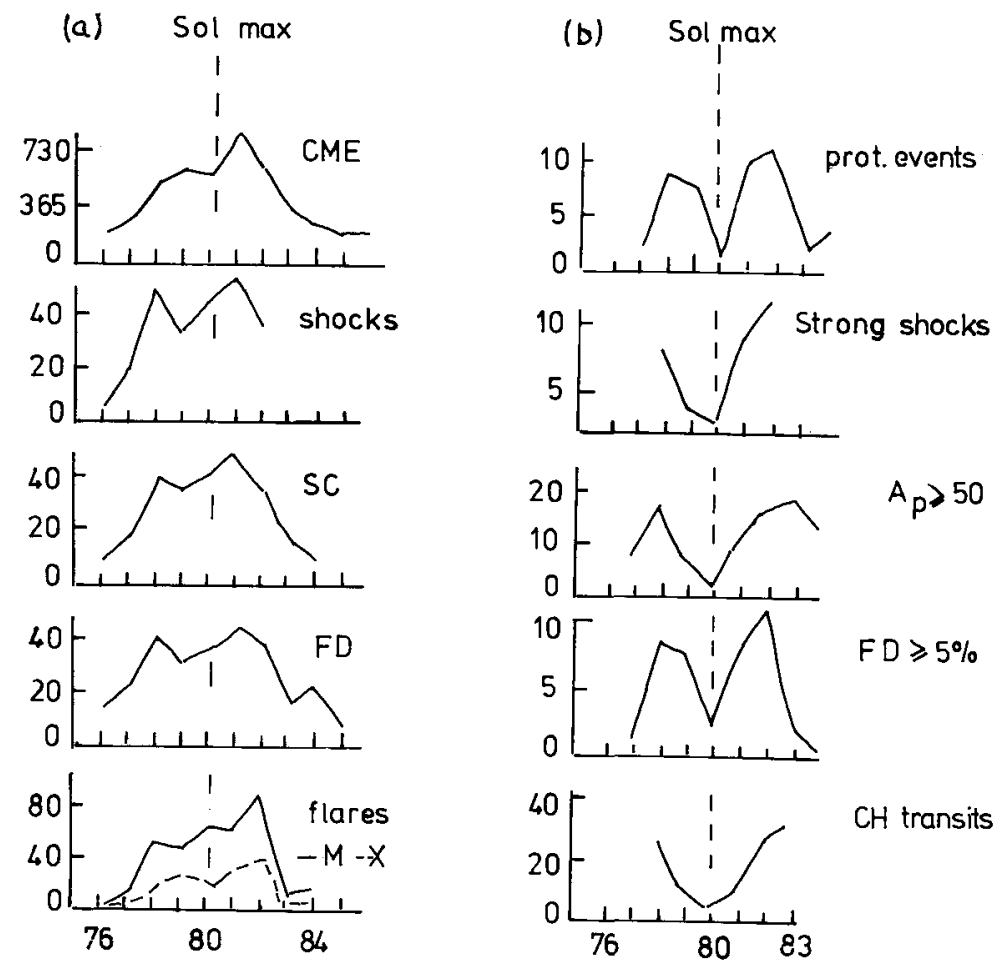

$\mathrm{CH}$ transits

Fig. 1. (a)Annual occurrence rates of CMEs, solar flares, typical interplanetary shocks, sudden commencement geomagnetic storms and Forbush decreases. (b) Annual occurrence rates for the strongest shocks, sudden commencements and geomagnetic storms; also for proton events (>10 MeV) and low latitude coronal holes.

ambient plasma. This implies a rather small mass for the compressed driver plasma and conservation of momentum then demands unrealistic initial speeds for CMEs leaving the Sun. A related problem is the frequent absence of rarefaction zones behind strong shocks, such zones being a necessary consequence of impulsive drivers. Possible sources of the sustained driver outflows are discussed.

\section{The absence of strong shocks during $\mathbf{1 9 8 0}$}

The Annual occurrence rates of CMEs, solar flares, interplanetary shocks, sudden commencement geomagnetic storms (SCs) and Forbush decreases (FDs) are shown in Figure 1(a). The data are from Webb \& Howard ${ }^{[3]}$, Krivsky et al. ${ }^{[4]}$ and Solar Geophysical Data. The occurrence rates of shocks, SCs and FDs follow that for CMEs quite well. By contrast, when only the 
(a)

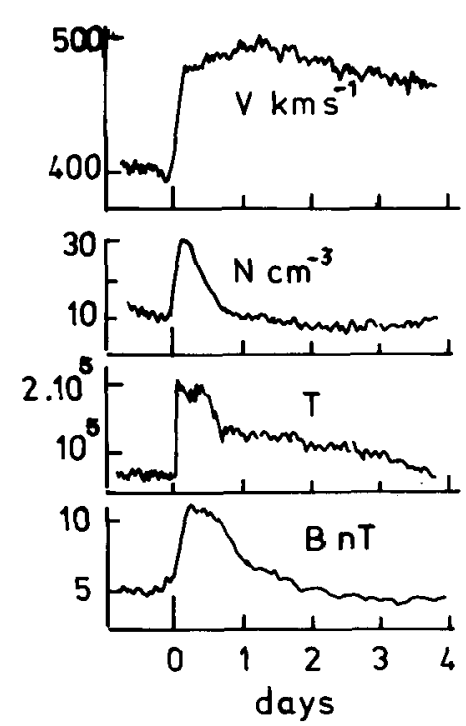

(b)
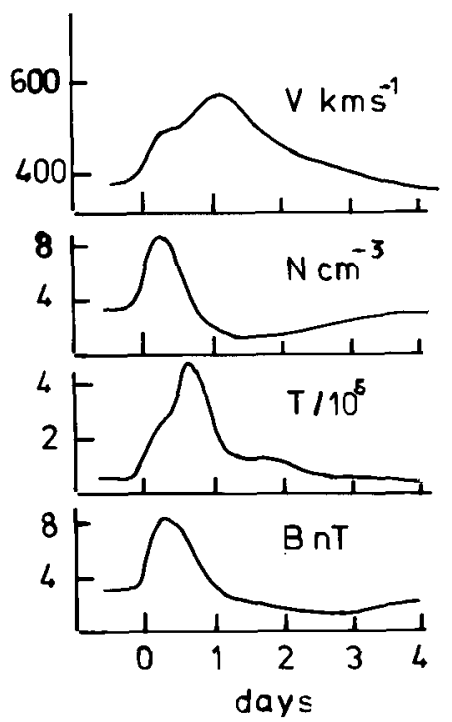

Fig. 2. (a) Average shock profiles observed near 1 AU. (b) Simulated profiles of an erupting solar wind stream.

strongest shocks are considered, the solar cycle variation is different and shows a notable absence of strong shocks at solar maximum. To define a strong shock consider the average behaviour for a sample of 103 shocks which occurred during 1972-79 as obtained by Borrini et al..$^{[1]}$ and shown in Figure 2 (a). Typical shocks exhibit a jump in wind speed of about $80 \mathrm{~km} \mathrm{~s}^{-1}$ and give Forbush decreases of about $2 \%$. I define a strong shock as one having a velocity inçrease $\geq 150 \mathrm{~km} \mathrm{~s}^{-1}$ which usually corresponds to a Forbush decrease $\geq 5 \%$. Using these criteria and identifying shocks in the nearly complete spacecraft data for $1978-82^{[5]}$ gives the occurrence rates shown in Figure 1(b) for strong shocks and large FDs. Also shown are corresponding data for major geomagnetic storms, proton events $(>10 \mathrm{MeV})$ and the transits of coronal holes.

The absence of strong shocks and shock-associated phenomena in 1980 is very striking and demands explanation. Similar, though less pronounced minima at solar maximum have been seen before in geomagnetic data ${ }^{[6]}$. Was there some reason why CMEs were so ineffective in causing strong shocks in 1980? Neither the properties of CMEs nor of the ambient solar wind show sufficient variation through the solar cycle to explain the 1980 minimum. It appears that strong shocks cannot be generated by CMEs alone and some other factor must be involved. The fact that low-latitude coronal 


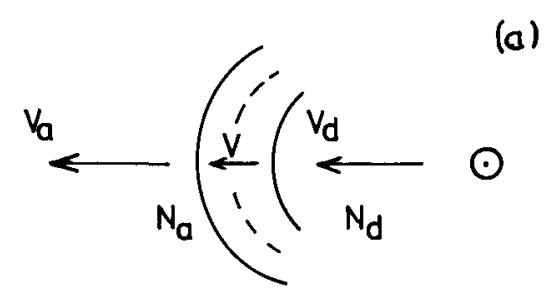

(b)

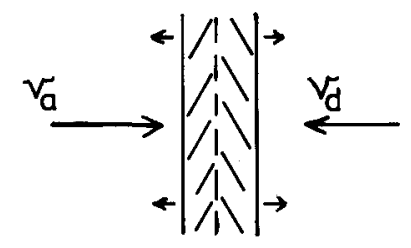

Fig. 3. (a) Schematic diagram of an interplanetary shock. (b) Planar shock model.

holes were also less frequent in 1980 is an interesting correlation that could be relevant.

\section{Mass and momentum relationships}

A very simple model will suffice to illustrate the main features of the shocked plasma in the compression zone and its development as the shock propagates. Only small deviations from radial motion are observed in the solar wind so it is a reasonable approximation to assume strictly radial velocities. The system is then effectively one-dimensional and a shock expanding with spherical symmetry about the Sun as shown in Figure 3(a) is equivalent to the planar shock in Figure 3(b). The dynamics will be considered in a frame of reference in which the contact discontinuity is at rest. Then with parameters as defined in Figure 3(b), and since the total momentum of the compression zone in this frame is zero we have,

$$
N_{a} v_{a}^{2}=N_{d} v_{d}^{2} .
$$

If $V$ is the instantaneous velocity of the contact discontinuity in the spacecraft frame of reference the transformed velocities are given by

$$
\begin{aligned}
& v_{a}\left(1+\sqrt{N_{a}} N_{d}\right)=v_{d}\left(1+\sqrt{N_{d}} N_{d}\right)=V_{d}-V_{a} \\
& 2 V=V_{a}+V_{d}+v_{a}-v_{d} .
\end{aligned}
$$


In the special case that $V_{a}$ and $V_{d}$ are constant and independent of radial distance $r$, and $N_{a}$ and $N_{d}$ both vary as $r^{-2}$, the total mass of the compression region increases at a uniform rate. For a strong shock, corresponding to a fourfold jump in density at the shock front, the Rankine-Hugoniot conditions require speeds of $v_{a} / 3$ and $v_{d} / 3$ for the forward and reverse shocks relative to the contact discontinuity. If $M_{a}$ and $M_{d}$ are the instantaneous masses of the compressed ambient and driver plasmas then using (1) we obtain

$$
M_{a} / M_{d}=\sqrt{N_{a} / N_{d}}
$$

Thus for a low density driver interacting with a higher density ambient plasma we have $M_{a}>M_{d}$ and vice versa. For a shock initiated at $r=r_{0}$ and observed at distance $R$, the total swept-up mass of ambient plasma $M_{a}(R)$ is

$$
M_{a}(R)=\left(R-r_{0}\right) N_{a}(R)\left(1-V_{a} / V\right) .
$$

The validity of this very simple model may be checked by comparing it with numerical simulations using a time-dependent MHD model. Such a model has been discussed by Smith \& Dryer ${ }^{[7]}$ who considered an 'erupting' high-speed stream abruptly injected into a uniform ambient wind and then sustained. The initial conditions at $r_{0}=0.08 \mathrm{AU}$ were $V_{a}=350 \mathrm{~km} \mathrm{~s}^{-1}$ and $V_{d}=750 \mathrm{~km} \mathrm{~s}^{-1}$. The corresponding plasma densities, translated to $1 \mathrm{AU}$, were $N_{a}=3 \mathrm{~cm}^{-3}, N_{d}=0.65 \mathrm{~cm}^{-3}$. The computed profiles of velocity, density and mass flux at $1 \mathrm{AU}$ are plotted in Figure 2(b). The velocity of the contact discontinuity is $\approx 475-500 \mathrm{~km} \mathrm{~s}^{-1}$ and the compression zone takes $\approx 25$ hours to pass an observer. The corresponding values derived for our simple model using (2) and (3) are $V=477 \mathrm{~km} \mathrm{~s}^{-1}, v_{a}=127 \mathrm{~km} \mathrm{~s}^{-1}$, $v_{d}=273 \mathrm{~km} \mathrm{~s}^{-1}$. Assuming strong shock conditions, the compression zone is expanding overall at a speed of $\left(v_{a}+v_{d}\right) / 3=133 \mathrm{~km} \mathrm{~s}^{-1}$, so that it has acquired a radial thickness of $\approx 0.25 \mathrm{AU}$ after travelling to $1 \mathrm{AU}$ and takes $\approx 22$ hours to pass an observer. The parameters derived from our model are therefore in good agreement with the MHD simulation.

When considering the total mass $\left(M_{a}+M_{d}\right)$ in the compression zone at $1 \mathrm{AU}$ it will be convenient to use the mass per unit area since the angular extent of interplanetary shocks is only known approximately. Integrating the mass flux for the MHD model (see Figure 2(b)) gives $\left(M_{a}+M_{d}\right) \approx$ $2 \times 10^{13}$ proton $\mathrm{cm}^{-2}$. Our simple model using (4) and (5) gives $M_{a}=$ $1.2 \times 10^{13}$ proton $\mathrm{cm}^{-2}$ and $M_{a} / M_{d}=2.1$ from which we obtain $\left(M_{a}+\right.$ $\left.M_{d}\right)=1.8 \times 10^{13}$ proton $\mathrm{cm}^{-2}$. Remembering that the value computed for the MHD model is not accurate to better than $\pm 10 \%$, since the boundary at the trailing edge of the compression zone is ill-defined, both models are again in reasonable agreement. 
For real interplanetary shocks $N_{a}$ and $N_{d}$ are unlikely to vary simply as $r^{-2}$, while $V_{a}$ and $V_{d}$ will not remain constant. Nevertheless, the model gives useful insights into real situations. For example, to model a shock driven impulsively by a CME, the ambient wind at, say, $0.1 \mathrm{AU}$ would be replaced by a high-speed driver for a few hours, and then returned to ambient conditions. The simple model would then be applicable so long as the driver is entering the compression zone. When the CME has fully entered the compression zone the compression zone continues to accumulate mass from the ambient plasma alone and begins to decelerate. Values of $M_{a}$ derived from (5) and $V$ observed at $1 \mathrm{AU}$ will then be underestimates since the mean transit speed must now exceed $V$. In this case a lower limit on the initial speed $V_{d}$ of the driver plasma near the Sun, obtained from conservation of momentum, is given by

$$
V_{d}(\text { initial })=V\left(M_{a} / M_{d}+1\right)-\frac{V_{a} M_{a}}{M_{d}}
$$

Real shocks are not necessarily driven impulsively all the way out to $1 \mathrm{AU}$. Interaction regions in which higher speed plasma tries to overtake slower plasma may generate compression waves which only steepen into shocks as the disturbance propagates. For example, an erupting stream need not start abruptly as assumed above; the speed could increase smoothly over some period. From elementary kinematics the velocity gradient required to produce a shock at distance $R$ is of the order $d V / d t>V^{2} / R$. For $V=$ $400 \mathrm{~km} \mathrm{~s}^{-1}$ and $R=1 \mathrm{AU}$ a velocity gradient exceeding $100 \mathrm{~km} \mathrm{~s}^{-1}$ per day should suffice. In this case the swept-up mass $M_{a}$ must, of course, be much less than the value given by equation (5) since the disturbance travels over most of the distance as a compression wave and not as a shock.

The signatures of strong interplanetary shocks are often too complex for simple analysis, but examples can be found when a single shock is superposed upon fairly stable ambient conditions and the simple theory is applicable. Two examples are shown in Figure 4. For the shock on 13 July 1982 using equation (5) we obtain $M_{a}=6.3 \times 10^{13}$ protons $\mathrm{cm}^{-2} \mathrm{~s}^{-1}$ and from the observed mass flux we have $\left(M_{a}+M_{d}\right)=1.0 \times 10^{14}$ protons $\mathrm{cm}^{-2} \mathrm{~s}^{-1}$ giving $M_{a} / M_{d}=1.7$. Equation (6) with $V=800 \mathrm{~km} \mathrm{~s}^{-1}$ and $V_{a}=550 \mathrm{~km} \mathrm{~s}^{-1}$ then gives $V_{d}$ (initial) $=1230 \mathrm{~km} \mathrm{~s}^{-1}$. This is near the upper limit of speeds for CMEs directly observed near the Sun and is not unreasonable but no account has been taken of deceleration which must increase $V_{d}$ (initial). For the shock of 16 November 1981 a similar analysis gives $V_{d}$ (initial) $>$ $1500 \mathrm{~km} \mathrm{~s}^{-1}$. These velocities are uncomfortably high. While it might be argued that the assumed density of the ambient plasma was actually less than that observed before and after the shock, thus reducing the estimated value of $M_{a}$ and hence decreasing $V_{d}$ (initial), the existence of a number of 

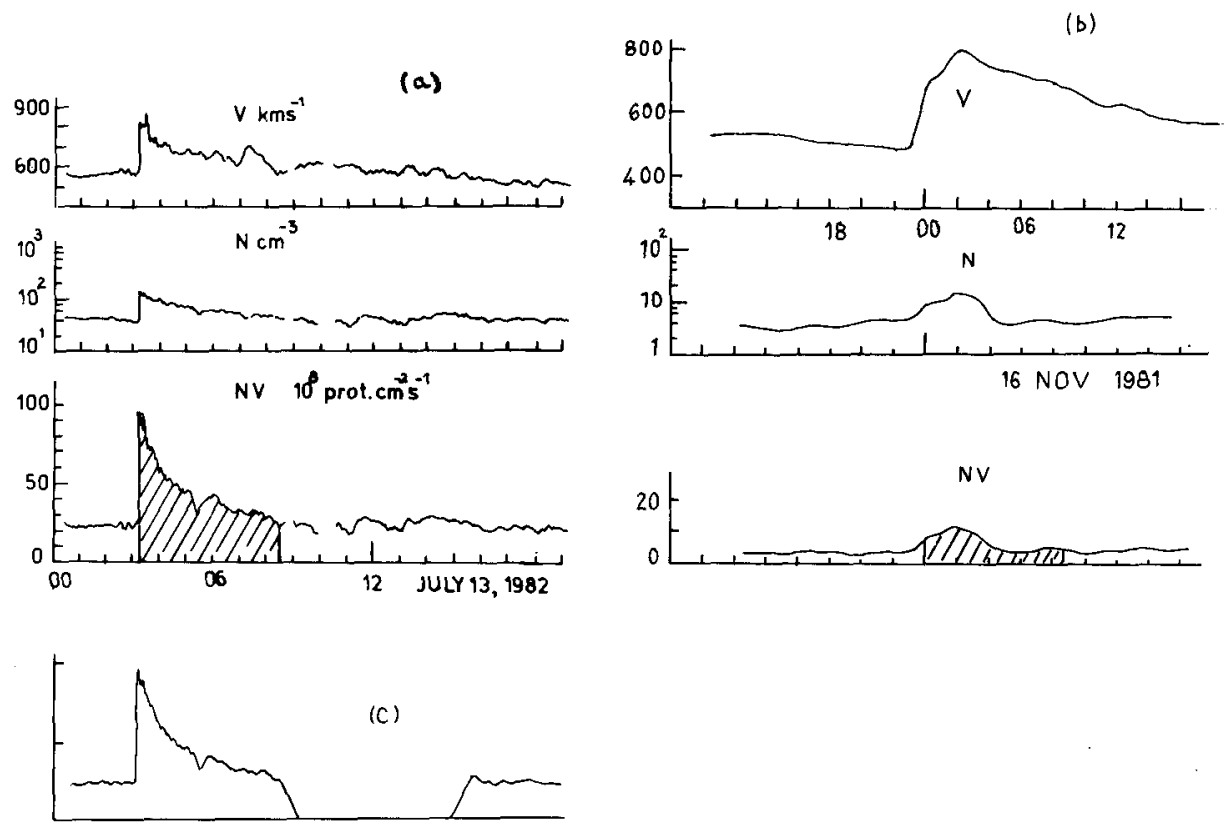

Fig. 4. Shock profiles including the mass flux of two strong shocks. Also shown schematically is the kind of rarefaction zone expected for a CME-driven shock.

similar cases raises doubts about the validity of an impulsive mechanism for these strong shocks.

Another problem for CME-driven shocks is the absence of rarefaction zones following the compression region. For example, a CME travelling at $600 \mathrm{~km} \mathrm{~s}^{-1}$ through an ambient wind of $400 \mathrm{~km} \mathrm{~s}^{-1}$ would be followed by a 'swept-out' depletion region of lower than ambient density which would last for about 24 hours at $1 \mathrm{AU}$. The numerical simulations of Smith \& Dryer ${ }^{[8]}$ show this effect very clearly. The kind of depletion zone required to compensate for the swept-up mass for the shock of $13 \mathrm{July} 1982$ is illustrated quantitatively in Figure 4(c). No depletion zone was observed for either shock and the average profiles shown in Figure 2(a) suggest that this is typical behaviour. If an impulsively generated shock was immediately followed by a new source of high speed wind which, fortuitously, had the same speed as the CME no depletion zone would be formed, but such an explanation of the observed profiles seems highly contrived. 


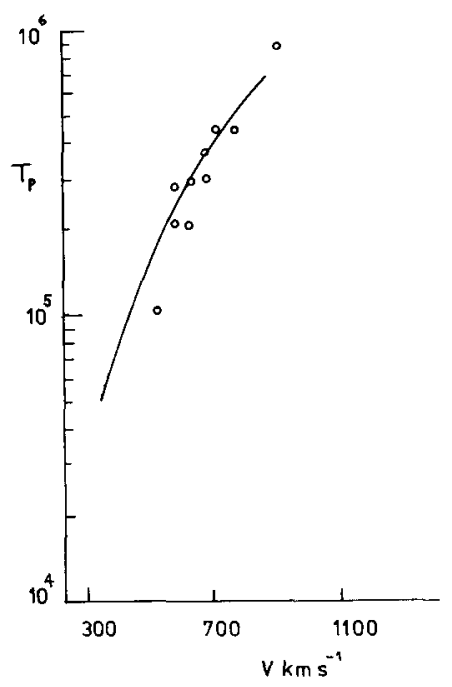

Fig. 5. Proton temperature versus velocity for driver plasma following a sample of strong interplanetary shocks.

\section{The nature of strong shock drivers}

The problems of initial speeds and the absence of depletion zones are avoided if strong shocks are generated by erupting streams and the simulated profiles in Figure 2(b) are very similar to observed shock profiles. The proton temperature and velocity of unshocked driver plasma following immediately behind the compression zones for a sample of strong shocks is shown in Figure 5, from which it is clear that the values are typical of those found in stable high speed streams. The fact that shocks are often followed by high speed outflows lasting for several days led Borrini et al. ${ }^{[1]}$ to suggest that CMEs could blow out previously closed magnetic field lines thereby creating new open-field regions which could act as new sources of high speed streams. In this case new coronal holes should be found near CME sites. While there is some evidence for the occurrence of small, transient coronal holes following CMEs Kahler \& Hundhausen ${ }^{[9]}$ have concluded that most of the area in the corona within CME boundaries remains closed or becomes so soon after the CME-eruption. It is therefore more reasonable to suppose that previously existing coronal holes are the source of erupting streams which subsequently persist for several days.

Since stable coronal holes at low latitudes are clearly not sources of strong shocks in the absence of solar activity the necessary conditions demand the presence of both coronal holes and active regions. Coronal holes often develop near active regions so a scenario in which the speed of the solar wind 
from a coronal hole is modulated in association with global disruptions of the magnetic field causing CMEs is not unlikely. Either an enhanced flux of Alfven waves from the chromosphere, or variations in the coronal magnetic field geometry in the acceleration zone of the wind could change the wind speed sufficiently to generate shocks. Alternatively CMEs might trigger the onset of erupting streams as suggested by Bravo et al. ${ }^{[10]}$

In addition to the evidence described in this paper it should be noted that imaging of interplanetary shocks using scintillation techniques has already shown that they are emitted from solar sources coincident with or close to low latitude coronal holes ${ }^{[11]}$. Overall, the evidence that the strongest shocks are driven by low-density, high-speed outflows emitted by coronal holes during periods of high solar activity is rather strong. Associated CMEs are likely to accompany such eruptions but are not, by themselves, sufficient causes of these shocks.

\section{References}

1. Borrini, G., Gosling, J.T., Barnes, S.J., and Feldman, W.C.: 1982, J. Geophys. Res., 87,4365 .

2. Kahler, S.W.: 1992, Annu. Rev. Astron. Astrophys., 30, 113.

3. Webb, D.F. and Howard, R.A.: 1994, J. Geophys. Res., 99, 4201.

4. Krivsky, L., Gopasyuk, S., and Soliman, M.A.: 1992, Publications Astr. Inst. Czechosl. No. 80.

5. Couzens, D.A. and King, J.H.: 1986, Interplanetary medium data book, supplement 3A NSSDC/WDC-A-R\&S 86-04, Natl. Space Sci. Data Cent. Greenbelt, MD.

6. Thompson, R.: 1990, Proceedings of Solar-Terrestrial Predictions Workshop, Leura, Australia Oct 16-20, 1989, Vol 1, 598.

7. Smith, Z., and Dryer, M.: 1991, Solar Phys., 131, 363

8. Smith, Z., and Dryer, M.: 1990, Solar Phys., 129, 387

9. Kahler, S.W. and Hundhausen, A.J.: 1992, J. Geophys. Res., 97, 1619

10. Bravo, S., and Perez-Enriquez, R.: 1994, Rev. Mexicana de Astr. y Astrophys., $28,17$.

11. Hewish, A., Tappin, S.J. and Gapper, G.R.: 1985, Nature, 314, 137. 